\title{
Investigation of dyspnea using cardiopulmonary exercise test among patients resorting to the respiratory tests
}

\author{
Seyedeh Zahra Fotook Kiaee, Besharat Rahimi
}

Advanced Thoracic Research Centre, Tehran University of Medical Science, Tehran, Iran

This article is distributed under the terms of the Creative Commons Attribution Noncommercial License (CC BY-NC 4.0) which permits any noncommercial use, distribution, and reproduction in any medium, provided the original author(s) and source are credited.

\begin{abstract}
Exertional dyspnea is a common complaint among patients which may be the final result of many diseases. Few researches have been conducted concerning the role of cardiopulmonary exercise test (CPET) in assessing unexplained dyspnea. Therefore, the aim of this study was a research on unexplained dyspnea using CPET among patients resorting to respiratory test unit of hospitals. This is a cross-sectional study that performed on 100 patients. With the direct supervision of experts, CPET was conducted using ergaspirometery device. The exercise data was interpreted by a pulmonologist and the results were classified into pulmonary diseases, cardiovascular diseases, obesity, deconditioning, and undetermined groups. 100 patients including 57 men and 43 women with an average age of 42 years and an average BMI of 28 and smoking frequency of $12 \%$ took part in this research. The following frequencies were reported for each complication: $24 \%$ for pulmonary diseases, $23 \%$ for cardiovascular diseases, $23 \%$ for deconditioning group, $15 \%$ for obesity, and $16 \%$ for the undetermined group. No significant difference was observed between these five groups in terms of gender distribution and smoking cigarette $(\mathrm{P}$-value $>0.05)$. In the age group older than 42 years, more patients were diagnosed with cardiac diseases and obesity. CPET may be really useful in determining the cause of unexplained dyspnea among most patients. Furthermore, the CPET can determine the presence or absence of disease, and if present, the nature of the limitation.
\end{abstract}

Key Words: Cardiopulmonary exercise test, unexplained dyspnea, pulmonary diseases, cardiovascular diseases.

Eur J Transl Myol 28 (3): 294-299, 2018

Dyspnea is a common problem in outpatient treatment centers. Exertional dyspnea is one of the most common complaints of patients which may be the end result of many diseases such as cardiovascular complications, pulmonary diseases, skeleton-muscular diseases, obesity, deconditioning, and psychological problems. ${ }^{1}$ In clinical assessment of patients' exertional dyspnea, stepwise diagnostic measures have not been clearly specified and the information we have regarding how to diagnose this complication is insufficient. Diagnosing dyspnea is relatively difficult as it is found in several diagnostic categories. These categories include background complications or more serious problems (such as cardiopulmonary complications) which need to be treated in the emergency service ward of hospitals. ${ }^{2,3}$ Timely diagnosis, measurement and treatment play a major role in controlling dyspnea. If physical examinations, chest radiography, and spirometry fail to diagnose dyspnea, other iagnostic tests such as cardiopulmonary exercise test (CPET) will be utilized. ${ }^{4}$ The main and most important application of cardiopulmonary exercise test is to determine the main causes of restrained activity and dyspnea. ${ }^{5,6}$ Moreover, unexplained exertional dyspnea or fatigue is a generally encountered problem in clinical practice and can pose a significant diagnostic challenge to physicians because the pathophysiology causing the exercise intolerance is not good understood. ${ }^{7-9}$ Few researches have been conducted concerning the role of CPET in assessing unexplained dyspnea, but it has been proven that CPET in such cases can limit differential diagnosis and sometimes introduce a single system as the restraining factor. ${ }^{10-12}$ Researchers believe that CPET among those patients with unexplained dyspnea can provide important and useful information for doctors. ${ }^{10}$ Another study showed the conclusion that submaximal cardiopulmonary exercise test is really useful in determining pulmonary causes and deconditioning among patients with cardiac failures. ${ }^{13}$ To determine the best modality in order to have a definite diagnosis, we may utilize this test. It is also used to realize if the 


\section{Dyspnea using cardiopulmonary exercise test}

Eur J Transl Myol 28 (3): 294-299, 2018

dyspnea the patient keeps complaining about has really restrained his activities or it is merely a subjective feeling. A completely normal CPET can reject existence of any significant diseases. CPET can help experts diagnose and measure dyspnea. This method is not without its limits. For instance, it fails to differentiate deconditioning from heart failure and more exclusive and accurate tests are required to determine and diagnose the main problem. ${ }^{14}$ CPET is able to evaluate these symptoms as it provides a general assessment of exercise capacity and defines the specific contributions of any cardiac, pulmonary mechanical, pulmonary vascular, hematologic, muscular, or neurologic limitations and also, CPET allows the simultaneous study of the responses of the cardiovascular and ventilator systems through the measurement and the integrative interpretation of a lot of parameters..$^{7-9}$ Considering the demographic condition and epidemiology of the common diseases observed in Iran, designing and conducting such researches to study the background causes of unexplained dyspnea seems quite necessary. Thus, the present research seeks to unexplained dyspnea using CPET among patients resorting to the pulmonary test unit of hospitals.

\section{Materials and Methods}

\section{Ethics}

The study was approved by the Institutional review board (IRB) of the Tehran University of Medical Science, and was performed in accordance with the Helsinki Declaration of 1964. All of the investigated subjects voluntary participated in our study and signed informed consent forms before enrollment.

\section{Participants and sampling}

This is a cross-sectional study conducted on patients resorting to the respiratory tests unit of Imam Khomeini Hospital of Tehran in 2015. In this study, we have assessed that a multidisciplinary approach that includes CPET dramatically decreases the time to diagnosis compared with traditional therapy and testing methods. With due observation of medical ethics principles and after obtaining patients' consent, they took part in the research.

\section{Inclusion criteria}

1. Patient's main complaint needs to be exertional dyspnea,

2. Patients whose initial examinations such as selfexpression, physical examination, electrocardiography (ECG), chest radiography, and spirometry had found no reason for their dyspnea;

3. Patients whose spirometry had found normal flowvolume loop, forced expiratory volume in one second (FEV1) $>8 \%$ predicted, and FEV1/ FVC (forced vital capacity) $>70 \%$.

4. Patients who were able to conduct cardiopulmonary test and finish it based upon the defined criteria.

\section{Exclusion criteria}

1. Acute cardiac infraction within the last one month, heart failure exacerbation, exertional syncope, uncontrolled arrhythmia, severe stenosis of the aortic valve, endocarditis, myocarditis, acute pericarditis,

2. Suspicion of dissecting aortic aneurysm, deep vein thrombosis (DVT) or acute pulmonary embolism,

3. Asthma or uncontrolled chronic obstructive pulmonary disease (COPD), O2sar $<85 \%$ while resting in room air, acute respiratory failure, acute pulmonary hypertension,

4. Stenosis of the main left coronary artery, more than $200 \mathrm{~mm} \mathrm{hg}$ systolic and $120 \mathrm{~mm} \mathrm{hg}$ diastolic blood pressure,

5. Hypertrophic cardiomyopathy, high degree heart block, severe electrolyte disorders, tachycardia and bradycardia arrhythmias, existence of nonadjustable

\section{Outcome assessment}

Based upon previous studies and with due observation of inclusion criteria, as many as 100 patients were selected to take part in the research. Early exercise termination would be prescribed by the expert if either chest pain, ischemic changes of ECG, hypotension, low $\mathrm{O} 2$ sat $<80 \%$, symptoms of respiratory failure, or diagnostic failures of brain were reported. A questionnaire containing items related to age, height, weight, body mass index (BMI), smoking cigarette, history of cardiac diseases and consuming medicines was used to collect primary patients' information. Ganshorn ergaspirometery device was used for cardiovascular exercise test. Using a cycle ergometer, the cardiopulmonary exercise test was conducted according to Incremental work rate principles. Doctors supervised how the test was conducted and any issue concerning patient's health and safety was recorded. The test results were interpreted by a pulmonologist and the data was classified into cardiovascular causes, pulmonary causes, obesity, deconditioning, and non-organic (undetermined) causes. When more than one factor was proposed as the cause of dyspnea in this classification, the factor with the greatest possibility of limiting the test was selected as the cause.

\section{Statistical analysis}

The resulting raw data was analyzed using SPSS v.19 and Kolmogorov-Smirnov Test was used to study data normality. Parametric statistical test of T-test and nonparametric statistical test of Chi-square test were used based upon the normality status. The level of statistical significance (P-value) in this research was set to less than 0.05 .

\section{Results and Discussion}

The present research was conducted on 100 patients undergoing CPET in Imam Khomeini Hospital in 2015. 43 patients were male and the remaining 57 were female. The patients' age ranged from 17 to 80 years old. 12 


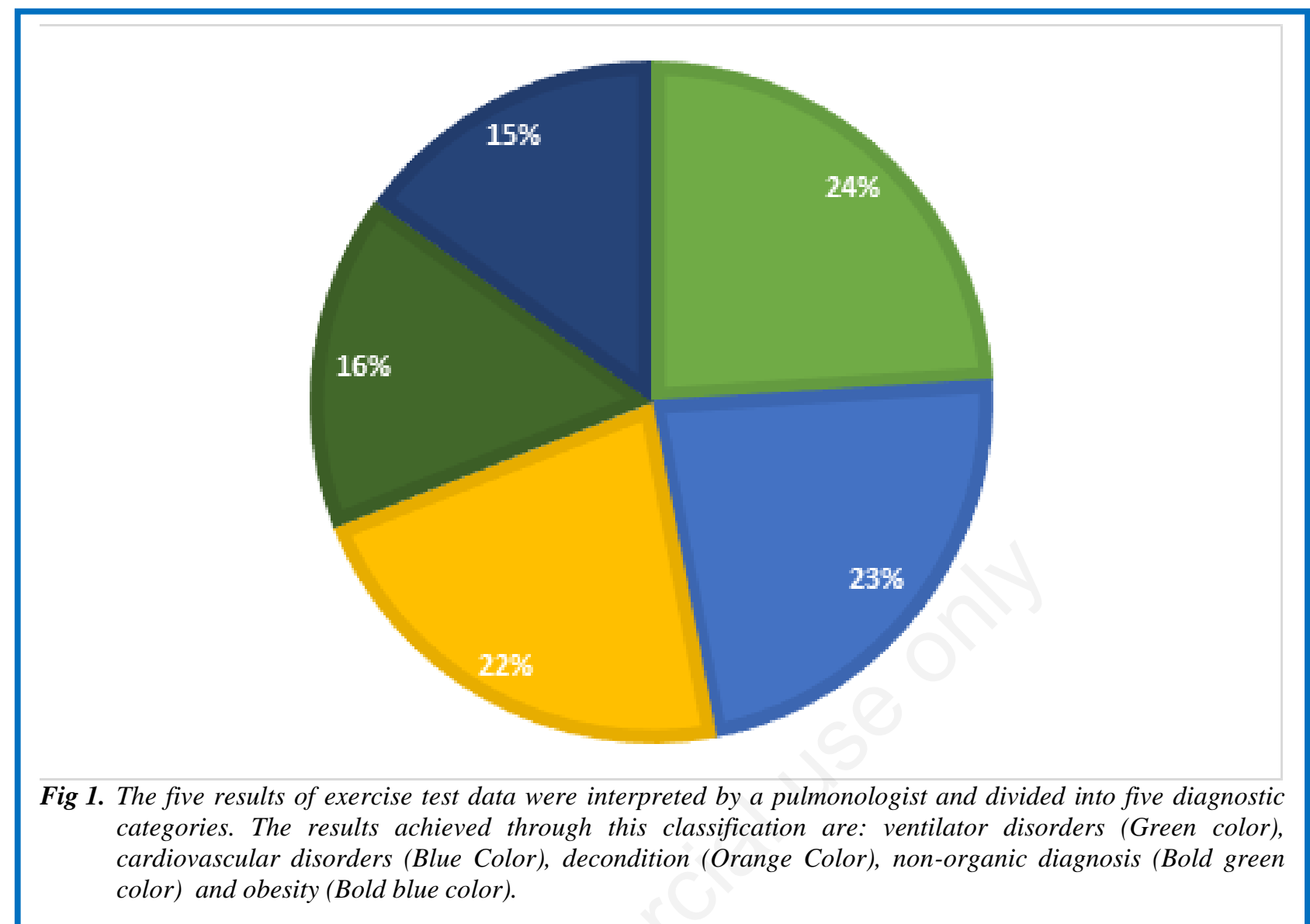

patients smoked cigarettes. Various studies pointed to the fact that the mean BMI of patients was 28. Exercise test data was interpreted by a pulmonologist and the results were divided into five diagnostic categories. The results achieved through this classification are as follows: 24 cases of ventilator disorders, 23 cases of cardiovascular disorders, 15 cases of obesity, 22 cases of decondition and 16 cases of non-organic diagnosis (Figure 1). A review of the correlation between these 5 results and people's age using ANOVA test indicated a significant correlation between these two variables $(\mathrm{P}$-value $=$ $0.0001)$. The highest and lowest means of exercise test results were reported for obesity $(51.80 \pm 10.31)$ and deconditioning $(35.77 \pm 10.48)$ respectively (Table 1$)$. The statistical comparison of exercise test results and patients' gender in the present research failed to show any significant difference between these variables (P-value $>$ 0.05) (Table 2). The patients were classified into two groups including those younger than and those older than 42 years. The statistical analysis indicated a statistically significant difference between these two groups (P-value $=0.007)$. A review of the frequency of smoking cigarettes indicated the following prevalence of smokers in each group: $16 \%$ in pulmonary group, $8.7 \%$ in cardiac group, $26.7 \%$ in obesity group, $4.5 \%$ in deconditioning group, and $6.3 \%$ in non-organic group. These results failed to show a significant difference between smoking cigarettes and exercise test results $(\mathrm{P}$-value $=0.244)$. In terms of the distribution of these 5 diagnoses of exercise test among people with BMI levels lower or higher than 30 after removing obesity variable as the most important factor of dyspnea from our analyses, no significant difference was observed among other diagnostic groups (P-value $>0.05)$ (Table 3$)$. The present study shows that cardiopulmonary exercise test can help diagnose the causes of dyspnea among those patients where routine tests have failed to explain their disease. ${ }^{15}$ When the 100 participants of our project took the cardiopulmonary exercise test, the following abnormalities were reported: 24 cases of ventilator failure, 23 cases of cardiovascular diseases, 15 cases of obesity, 22 cases of deconditioning, and 16 cases of non-organic diagnosis (normal cardiopulmonary exercise test). The number of patients diagnosed with more than one problem in exercise test was quite limited (5 cases). Using self-expression, examinations and exercise test results and, in some cases, auxiliary methods such as echocardiography and Methacholine test, we tried to choose a more probable diagnosis in order to make the classifications and conclusions more homogenous. Higher cases of obesity as the cause of dyspnea were observed among women, but this factor did not lead to significance between the parameters of gender and exercise test results. Various studies showed that smoking cigarettes could not make a significant difference among the five groups. The results of studying BMI levels above or lower than 30 among the 
Table 1: The correlation between exercise test results and patients' age

\begin{tabular}{|c|c|c|c|c|}
\hline \multirow{2}{*}{ Diagnosis } & \multicolumn{3}{|c|}{ Age } \\
\cline { 2 - 3 } & Mean & Std. Deviation & P-Value & F \\
\hline Poulmonary & 37.9583 & 14.73676 & 0.0001 & \multirow{2}{*}{6.177} \\
\hline Cardiac & 48.9565 & 11.28345 & \\
\hline Obesity & 51.8000 & 10.31781 & \\
\hline Deconditioning & 35.7727 & 10.48778 & \\
\hline Non Organic & 42.3750 & 13.60821 & \\
\hline
\end{tabular}

$* * * * * * * * * * * * * * * * * * * * * * * * * * * * * * * * * *$

Table 2. A comparison between patients' gender and the five results of exercise test

\begin{tabular}{|c|c|c|c|c|c|c|c|}
\hline \multicolumn{2}{|c|}{} & \multicolumn{5}{c|}{ Diagnosis } & \multirow{2}{*}{ P-Value } \\
\cline { 3 - 7 } \multicolumn{2}{|c|}{ Sex } & Poulmonary & Cardiac & Obesity & Deconditioning & Non Organic & \\
\cline { 2 - 7 } & Female & 12 & 10 & 3 & 11 & 7 & 0.384 \\
\hline
\end{tabular}

$* * * * * * * * * * * * * * * * * * * * * * * * * * * * * * * * * *$

Table 3. Comparing people's BMI and the results of exercise test

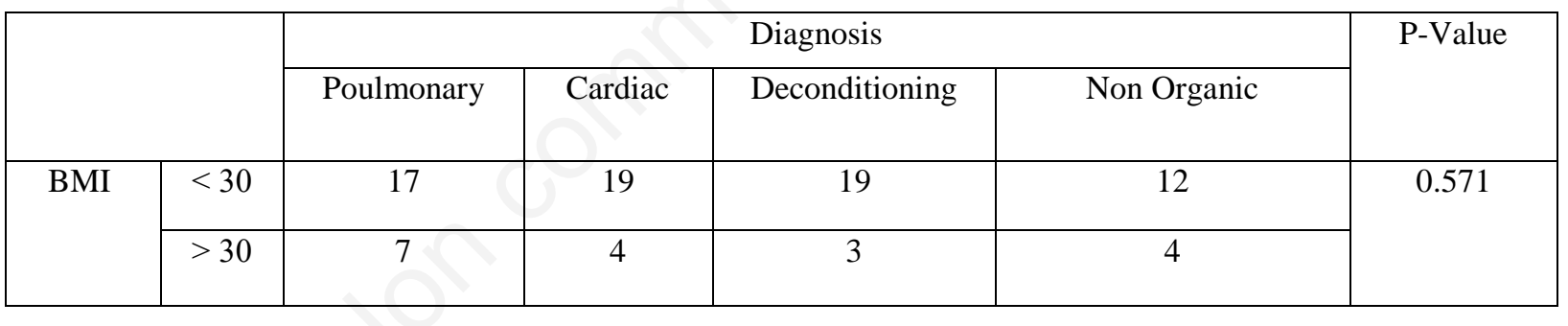

five groups (obesity as the most important variable of dyspnea was removed) showed no significant difference between these factors. A higher rate of cardiac diseases was reported among those older than 42 years, while higher rates of pulmonary diseases and deconditioning were reported among those younger than 42 . This field of research abounds with many ambiguities where many researches have been conducted mostly dealing with the etiology of dyspnea. Despite superficial differences, the results reported in such studies exhibit a rational alignment and general agreement. A comprehensive and general assessment of the present research shows a general agreement and alignment between it and other researches in this field despite superficial differences. For instance, a research was conducted on 50 patients that 7 cases of cardiac restrictions, 17 cases of pulmonary restrictions, 14 cases of obesity or deconditioning and 16 cases of dyspnea were observed. 5 patients were diagnosed with more than one clinical diagnosis. Normal FEV1 and FVC were the criteria used in this research. They arrived at the conclusion that most patients with chronic dyspnea could be diagnosed trough CPET. ${ }^{16}$ In a similar retrospective research by Simons et al., the patients resorting to a tertiary lung center in the Netherlands in order to examine their unexplained dyspnea took part in a study. These researchers used incremental protocol with cycle ergometer to reach symptom-limitation. This research was conducted on 40 patients. 55\% of them had resorted to therapeutic centers due to unexplained dyspnea, while the remaining $45 \%$ were complaining of dyspnea disproportionate to their cardiopulmonary state. The cardiopulmonary test in this research could highlight the restricting factors of patients' activities. They pointed to obesity, cardiac 
diseases, and hyperventilation syndrome as the most common causes of dyspnea. They finally arrived at the conclusion that using cardiopulmonary exercise test as a diagnostic assessment method is very useful in determining the nature of these patients. ${ }^{17}$ Another study was conducted by Webber et al. on 72 patients whose self-expression, examinations, chest radiography and spirometry had done no good in diagnosing the cause of their dyspnea. Using standard diagnostic assessments for 58 patients helped identify the cause of dyspnea but no cause of dyspnea was found for 14 patients. ${ }^{18,19}$ In terms of the number of patients studied (sample size), the number of cases diagnosed and the ability to differentiate between various diagnoses, our research is similar to previous researches and sometimes even better than those described above.

In conclusion, CPET is capable of diagnosing the cause in most cases and it may be used as a roadmap to determine more complete and advanced diagnostic modalities. Furthermore, CPET can determine the presence or absence of diseases, and, if present, the nature of the functinal limitations.

\section{List of acronyms}

CPET - cardiopulmonary exercise test,

DVT - deep vein thrombosis,

ECG - electrocardiography,

FEV1 - forced expiratory volume in one second,

FVC - forced vital capacity,

COPD - chronic obstructive pulmonary disease,

BMI - body mass index

\section{Author's contributions}

Each author contributed in equal part to the manuscript.

\section{Acknowledgments}

Funding: None.

This research received no specific grant from any funding agency in the public, commercial or not-for-profit sectors.

\section{Conflict of Interest}

The authors declare no conflicts of interests.

\section{Ethical Publication Statement}

We confirm that we have read the Journal's position on issues involved in ethical publication and affirm that this report is consistent with those guidelines.

\section{Corresponding Author}

Besharat Rahimi, Advanced Thoracic Research Centre, Tehran University of Medical Science, Tehran, Iran. Phone: +989128589591

Email: Besharatrahimi@yahoo.com

E-mail of co-author

Seyedeh Zahra Fotook Kiaee: Zahra.f.kiaee@gmail.com

\section{References}

1. Parshall MB, Schwartzstein RM, Adams L, et al. An official American Thoracic Society statement: update on the mechanisms, assessment, and management of dyspnea. Am J Respir Crit Care Med 2012;185:435-52

2. Tobin DL, Reynolds RVC, Holroyd KA, et al. SelfManagement of Chronic Disease: Handbook of Clinical Interventions and Research. 1986. New York: Academic Press 1986:21-48

3. Lorig KR1, Sobel DS, Stewart AL, et al. Evidence suggesting that a chronic disease self-management program can improve health status while reducing hospitalization: a randomized trial. Med Care 1999;37:5-14.

4. Neder JA, Fuld JP, Overend T, et al. Effects of formoterol on exercise tolerance in severely disabled patients with COPD. Respir Med 2007;101:2056-64.

5. Balady GJ, Arena R, Sietsema K, et al. Clinician's Guide to cardiopulmonary exercise testing in adults: a scientific statement from the American Heart Association. Circulation 2010;122:191-225.

6. Myers J. Essentials of cardiopulmonary exercise testing. Human Kinetics Publishers, 1996.

7. Huang W, Resch S, Oliveira RK, et al. Invasive cardiopulmonary exercise testing in the evaluation of unexplained dyspnea: Insights from a multidisciplinary dyspnea center. Eur J Prev Cardiol 2017;24:1190-9.

8. Toma N, Bicescu G, Enache R, et al. Cardiopulmonary exercise testing in differential diagnosis of dyspnea. Maedica (Buchar) 2010;5:214-8.

9. Datta D, Normandin E, ZuWallack R. Cardiopulmonary exercise testing in the assessment of exertional dyspnea. Ann Thorac Med 2015; 10:77-86.

10. Service FJ. Hypoglycemia, including hypoglycemia in neonates and children. In: Degroot LJ et al. Endocrinology, 3th edition. Philadelphia. WB Saunders 1995;1605-6.

11. Askanazi J, Nordenstrom J, Rosenbaum SH, et al. Nutrition for the patient with respiratory failure: glucose vs. fat. Anesthesiology 1981;54:373-7.

12. Schmidt RF, Thews G. Human physiology. 2nd edition. London. Publisher, Springer-Verlag, 1989.

13. Askanazi J, Rosenbaum SH, Hyman AI, et al. Respiratory changes induced by the large glucose loads of total parenteral nutrition. JAMA 1980;243:1444-7

14. Albouaini $\mathrm{K}$, Egred $\mathrm{M}$, Alahmar $\mathrm{A}$, et al. Cardiopulmonary exercise testing and its application. Heart 2007;93:1285-92.

15. Ferrazza AM, Martolini D, Valli G, et al. Cardiopulmonary exercise testing in the functional and prognostic evaluation of patients with pulmonary diseases. Respiration 2009;77:3-17. 


\section{Dyspnea using cardiopulmonary exercise test}

Eur J Transl Myol 28 (3): 294-299, 2018

16. Arena R, Sietsema KE. Cardiopulmonary exercise testing in the clinical evaluation of patients with heart and lung disease. Circulation 2011;123: 66880.

17. Defronzo RA, Ferrannini E. Regulation of intermediary metabolism during fasting and feeding. In: DeGroot LJ et al. Endocrinology, 3rd edition, Philadelphia. WB Saunders 2015;1391410.

18. Bhatt DV, Kocheril AG. Submaximal cardiopulmonary exercise testing for the evaluation of unexplained dyspnea. South Med J 2014;107:144-9.

19. Webber J, Macdonald I. The cardiovascular, metabolic and hormonal changes accompanying acute starvation in men and women. Br J Nutr 1994;71:437-47.

Submission: May 14, 2018

Revision received: May 28, 2018

Acceptance: May 29, 2018 